\title{
Nuevas políticas, nuevas miradas y metodologías de evaluación. ¿Cómo evaluar el retorno social de las políticas culturales?
}

\author{
Nicolás Barbieri \\ Adriana Partal \\ Eva Merino \\ Universitat Autònoma de Barcelona \\ nicolas.barbieri@uab.cat
}

Recibido: 23-06-2009

Aceptado: 30-06-2010

Cuando el saber se especializa, crece el volumen total de la cultura. Ésta es la ilusión y consuelo de los especialistas

(En Juan de Mairena, de Antonio Machado)

\section{Resumen}

El cambio en los objetivos y en los instrumentos de intervención de las políticas públicas no siempre comporta el desarrollo de nuevas perspectivas y metodologías para su evaluación. El caso de las políticas culturales ejemplifica esta realidad. Por un lado, han adquirido un rol fundamental en el desarrollo territorial que busca integrar una economía del conocimiento con la cohesión social, la gobernanza y la sostenibilidad. Sin embargo, su perspectiva de evaluación continúa centrada en criterios estéticos, de consumo cultural o, simplemente, en las externalidades de la cultura. Así, este artículo contribuye al desarrollo de metodologías para evaluar de forma científica el retorno social de las políticas culturales, su valor público y los beneficios producidos para la ciudadanía.

Palabras clave: políticas públicas; evaluación de políticas; políticas culturales; valor público de la cultura.

Abstract. New Policies, New Perspectives, and Methodologies of Evaluation: How to Evaluate the Social Return of Cultural Policies?

Change in policy objectives and instruments does not always entail the development of new perspectives and strategies for policy evaluation. Cultural policies exemplify these circumstances. On the one hand, they have achieved a crucial role in the territorial development which tries to integrate knowledge economy with social cohesion, governance, and

Queremos agradecer a Joan Subirats Humet y Xavier Fina Ribó, directores de la investigación «El retorno social de las políticas culturales», realizada con la colaboración del Departament de Cultura de la Generalitat de Catalunya. 
sustainability but on the other hand, their policy evaluation perspectives remain focused on aesthetic and cultural consumption criteria, or simply in culture externalities. Thus, this article contributes to the development of methodologies that evaluate scientifically the social return of cultural policies, their public value and benefits for citizens.

Key words: public policy; policy evaluation; cultural policy; public value of culture.

\section{Sumario}

1. Introducción: conceptos clave y objetivos

Una mirada del retorno social: el acento en las externalidades de la cultura

Límites de la perspectiva instrumental del retorno social de las políticas culturales

Nuevas miradas sobre el retorno social de las políticas culturales
Nuevas perspectivas, ¿nuevos indicadores?

Nuevos indicadores, ¿antiguos problemas?

Una propuesta de doce índices del retorno social de las políticas culturales

Consideraciones finales

Referencias bibliográficas

\section{Introducción: conceptos clave y objetivos}

Analizar el campo de las políticas públicas culturales implica enfrentarse, en primer lugar, a un reto conceptual. Delimitar, aunque solo sea de forma operacional, el campo de la cultura y el de las políticas públicas que se pretende estudiar es enfrentarse con palabras que nacen y evolucionan en situaciones cambiantes relacionadas con el ejercicio del poder. Cada aplicación del término cultura (y del de política cultural) implica su redefinición, y el desgaste facilita su utilización poco rigurosa y a veces incluso demagógica. Definir aquello que es intrínsecamente histórico es tarea condenada a la caducidad, pero esto no puede implicar la renuncia a conseguir coherencia en las estructuras teóricas y un cierto control conceptual sobre diferentes ámbitos de la realidad, siempre que se reconozcan sus limitaciones y debilidades.

La política cultural se refiere a los soportes institucionales que canalizan tanto la creatividad estética como los estilos colectivos de vida: es (o mejor, quiere ser) un puente entre los registros estético y antropológico (Miller y Yúdice, 2004). Si bien se trata de una herramienta mucho más compleja que la idea de algunos artistas empleados por el rey en la corte, no es hasta el siglo XX que organismos como la UNESCO se abocan a formular una definición de cultura y a controlar conceptualmente el espacio de la política cultural. La cultura se identifica entonces con «el conjunto de las artes y las letras, los modus vivendi, los derechos humanos fundamentales, los sistemas de valores y las creencias de una sociedad o grupo social» (UNESCO, 1982: 1). Se acentúa, así, progresivamente el papel de las políticas culturales, que se definen, por un lado, como un instrumento de promoción de bienes y servicios culturales (cultura en sentido pragmático) y, por otro, como herramienta que puede transformar las relaciones sociales, dar soporte a la diversidad e incidir en la vida ciudadana (visión valorativa de la cultura). 
Ahora bien, para comprender la evolución del campo de acción de las políticas culturales, puede resultar de utilidad observar los objetivos que se le han reconocido tradicionalmente y las variaciones en este sentido. Se trata de un primer paso que resulta fundamental para la construcción coherente y precisa de los criterios de evaluación de dichas políticas.

Tras la Segunda Guerra Mundial, con el reto común de la reconstrucción nacional y cultural que presentan muchos estados, la política cultural adquiere, en el mundo occidental, un carácter institucional significativo. Mientras el ámbito anglosajón consolida una política pública centrada en los incentivos fiscales, en un sistema basado en el principio del arm's lenght (distancia que busca impedir la injerencia gubernamental en la gestión del apoyo a las artes y la cultura), el modelo continental europeo encabezado por Francia se vuelca a la intervención directa de la Administración. El principio de democratización de la cultura guía la mayoría de políticas de los estados de bienestar, y la intervención gubernamental se centra en el fomento de la libre creación artística, la promoción de la oferta cultural de calidad (y del acceso a ella) y la protección del patrimonio.

A estos modelos se sumarán las políticas públicas de fomento de la democracia cultural, con la promoción de espacios de participación y expresión social. Tiempo después, con la consolidación del concepto de industrias culturales, la acción de gobierno buscará apoyar a la actividad artística y profesional, a los creadores y al desarrollo de los llamados sectores culturales. De forma complementaria, otro objetivo prioritario será el estímulo de la demanda cultural. Por lo tanto, el consumo cultural se consolida como indicador del desarrollo cultural de la sociedad e incluso, en algún caso, como criterio de evaluación de las políticas públicas.

En definitiva, reconociendo la capacidad y la legitimidad de las políticas culturales para contribuir a la mejora de las capacidades creativas estéticas de las personas, a la profesionalización y consolidación de los sectores de las artes y la cultura, así como su aportación al desarrollo económico, los cambios recientes en los objetivos que parecen asumir las políticas culturales vuelven necesario ampliar la perspectiva de análisis. Por eso este artículo identifica los aspectos más importantes del proceso de expansión del campo de actuación de las políticas culturales. Si diferentes actores esperan que las políticas culturales se integren en la promoción de diferentes objetivos (cohesión social, interculturalidad, educación, regeneración urbana, participación política y gobernanza, seguridad y paz, sostenibilidad, etc.), resulta lógica la necesidad de replantear los modelos de evaluación basados en criterios estrictamente de eficiencia económica y/o en base a criterios estéticos y de consumo cultural.

Pero esta tarea no puede afrontarse sin un análisis ni una valoración previa de la incidencia real de la acción cultural en la mejora de la calidad de vida de las personas, los beneficios para la ciudadanía y la contribución al desarrollo de sociedades cohesionadas y sostenibles. ¿En qué esferas de la realidad social y, sobre todo, de qué manera repercuten de forma significativa las accio- 
nes culturales que promueven las políticas públicas? ¿Cuál es el valor público que añaden? ¿Como se puede medir este impacto?

Este artículo pretende generar conocimiento útil sobre estas cuestiones y avanzar en la creación de instrumentos para diagnosticar el rendimiento de las políticas culturales públicas. Para ello, se parte del análisis de la perspectiva sobre el retorno social de las políticas culturales que, en buena medida, se ha ido consolidando desde la década de 1980. Se advierte, tras dicho análisis, que un énfasis desmesurado en las externalidades de la cultura y una consecuente dirección acrítica en la evaluación de las políticas culturales pueden resultar contraproducentes para su legitimidad y capacidad de incidencia. A continuación, se busca contribuir a la construcción de una mirada alternativa sobre el retorno social de las políticas culturales y a la mejora en los criterios de su evaluación. Se presentan nueve ejes para describir el valor público de las acciones de las organizaciones culturales, outcomes, no necesariamente vinculados a categorías instrumentales. Finalmente, tras una revisión del estado actual de la cuestión sobre los indicadores de políticas culturales a nivel internacional, se advierte y se ejemplifica sobre algunas de las tendencias más nocivas en la utilización de indicadores en las políticas públicas. Teniendo en cuenta que la construcción de indicadores no es una tarea únicamente técnica, se elabora una propuesta que incluye un conjunto de índices considerados como más significativos y apropiados para dar cuenta del retorno social de las políticas culturales. Se incluye también una reflexión sobre los criterios para poder medir la calidad y la envergadura de los indicadores en la evaluación de políticas públicas.

Como apunte metodológico, este análisis parte de la comparación de casos internacionales significativos en el estudio del retorno social de las políticas culturales. Para ello, se ha realizado un trabajo de análisis documental a partir de la búsqueda de fuentes publicadas en un ámbito internacional, incluyendo bibliografía científica, informes y presentaciones de experiencias significativas desarrolladas por diferentes instituciones, así como sistemas de indicadores y bases de datos disponibles. Esta revisión bibliográfica se ha realizado teniendo en cuenta los ámbitos sectoriales de incidencia de las políticas culturales en la sociedad (educación, salud, bienestar social, medio ambiente, etc.), pero también, y fundamentalmente, los atributos y los valores que las acciones culturales promovidas por las políticas culturales contribuyen a desarrollar en la ciudadanía.

El alcance de nuestro análisis y nuestra propuesta depende, lógicamente, de las distintas tradiciones de políticas culturales y, sobre todo, del nivel de desarrollo que tenga en cada país la implementación de políticas culturales y la reflexión y la evaluación sobre su retorno social. Ahora bien, este artículo tiene como objetivo producir un tipo de conocimiento generalizable de forma limitada (Ragin, 1987) a un tipo de política pública. Por un lado, cabe destacar que los modelos clásicos de políticas culturales comienzan a resultar caducos e inadecuados para atender a las expectativas y resolver los dilemas actuales, por lo que sus límites se desdibujan y dan paso a nuevas configuraciones institu- 
cionales. Y, por otro, si bien el análisis del retorno social de las políticas culturales ha venido despertando cada vez más atención, todavía es una tarea relativamente inédita. En este sentido, la utilización de fuentes mayoritariamente anglosajonas en nuestro estudio es el reflejo de la preeminencia de ese origen, tanto en la implementación de políticas como en su análisis científico. Se trata de una decisión basada en la disponibilidad de información y su alcance analítico, pero también en una valoración de la relevancia empírica de las políticas analizadas.

\section{Una mirada del retorno social: el acento en las externalidades de la cultura}

Las primeras referencias documentadas sobre el análisis del impacto social de las políticas culturales se remontan a mediados de la década de 1980. A partir de ese momento, tanto las experiencias de implementación de políticas públicas como los estudios referidos a ese tipo de impacto han ido ganando más importancia y relevancia para los agentes implicados.

La perspectiva que han adoptado las políticas que intentan poner de manifiesto la importancia de la acción cultural para el desarrollo de las sociedades, pero también la de muchas de las investigaciones científicas sobre esta cuestión, han centrado su atención en la externalidades de la cultura. Se enfatiza la supuesta capacidad de las políticas culturales para contribuir al desarrollo de los objetivos de otras políticas públicas sectoriales, para facilitar la consecución de los objetivos de la acción pública en otros ámbitos y agendas: educación, salud, medio ambiente, seguridad, urbanismo, etc. Se defiende y se argumenta así en favor de la función instrumental de la acción cultural que recibe apoyo por parte de la Administración pública. Para analizar esta perspectiva, presentamos de forma sintética tres ámbitos de políticas públicas significativos, y a la vez diversos, en lo que respecta a los vínculos con las políticas culturales y su impacto social. Un sector tradicionalmente vinculado a las políticas culturales (educación), un campo que, desde su consolidación, ha establecido puentes con el ámbito de la cultura (medio ambiente) y, finalmente, un ámbito (salud) que se puede considerar más reciente en lo que se refiere al retorno social de las políticas culturales.

En primer lugar, resulta extensa la bibliografía (sobre todo anglosajona) que analiza las aportaciones de la cultura al ámbito de la educación, así como las iniciativas políticas desarrolladas en este sentido. Los vínculos entre estos dos ámbitos se presentan generalmente bajo la idea que el arte y la cultura pueden contribuir al desarrollo de capacidades y competencias personales, profesionales y laborales de las personas; por ejemplo: las oportunidades generadas en los programas educativos de formación continuada a lo largo de la vida (Life Long Learning) o la creatividad y las competencias interpersonales adquiridas a través de la participación en actividades culturales. Por su parte, se señala la aportación del arte y la cultura como un elemento más en la ampliación y la mejora del sistema educativo. Casi identificado como contrapartida, se argumenta que las experiencias educativas vinculadas con estos campos generan, a 
largo plazo, un incremento del interés en las artes y la cultura por parte del colectivo de estudiantes ${ }^{1}$.

En segundo lugar, cabe destacar que las políticas culturales han vinculado parte de sus objetivos e instrumentos de intervención a aquellos planteados desde las políticas medioambientales. La acción cultural ha significado una contribución a la consecución de objetivos planteados desde el ámbito de la promoción del medio ambiente, y especialmente la noción de sostenibilidad vinculada a la de ecología. De forma explícita, se excluye de esta sección el debate sobre las relaciones entre el desarrollo de políticas culturales y lo que se ha convenido en llamar "procesos de regeneración urbana", sobre el que volveremos en los apartados finales del artículo.

En cualquier caso, resulta importante remarcar que la idea de sostenibilidad cultural ha ido cobrando cada vez más relevancia, tanto en la literatura académica internacional como en muchas de las iniciativas de políticas públicas culturales $^{2}$. Al menos, se identifican tres dimensiones con las que se vincula el concepto de sostenibilidad cultural: económica, social y tecnológica (Jeannotte, 2008). Lógicamente, si nos centramos en el impacto social de las políticas culturales que adoptan esta estrategia, la dimensión social resulta la más destacable. En este sentido, uno de los autores más reconocidos y adoptados en buena parte por las políticas culturales en Australia es Jon Hawkes (2001). En su modelo de desarrollo sostenible, la vitalidad cultural es un elemento central y estratégico. Se trata del cuarto pilar (conjuntamente con la equidad social, la responsabilidad medioambiental y la vitalidad económica) necesario para conseguir un desarrollo sostenible de las sociedades.

Finalmente, el ámbito de la salud ha adquirido una importancia creciente en iniciativas públicas y en los estudios sobre el impacto social de las políticas culturales. Nuevamente, la gran mayoría desarrolladas en países anglosajones. Así, la bibliografía académica (Matarassso, 1997; Cave y Couts, 2002; Mills y Brown, 2004; Duxbury et al., 2007) ha propuesto como retorno social de la acción promovida por las políticas culturales la mejora integral de la salud física, mental y psíquica de las personas. Este hecho se concreta, según el caso analizado, en diferentes aspectos y niveles: en las mejoras en las infraestructuras de asistencia sanitaria, en los tratamientos médicos o, directamente, en los resultados finales sobre la salud de las personas. Por su parte, diferentes investigadores (Barraket, 2005; Matarasso, 1997; Mills y Brown, 2004) han identificado, sobre todo en el Reino Unido, Canadá y Australia, un interés cre-

1. Son casos significativos, en este sentido, las políticas públicas (y particularmente las locales) de Inglaterra, Irlanda y Escocia, con investigaciones y programas específicos impulsados por sus consejos de las artes (arts councils). Se puede encontrar una descripción y un análisis en Ruiz (2004) o Scottish Arts Council (2008).

2. Este tipo de enfoques está presente en anàlisis de políticas culturales implementadas en Nueva Zelanda, Australia, Canadà o Escocia. Desde diferentes puntos de vista, se estudian los efectos del arte público sobre el territorio (Morris y Cant, 2004), el impulso a corrientes de artistas como la denominada eco-arts (Duxbury et al., 2007) o el papel de la cultura en estrategias de desarrollo sostenible (Mills y Brown, 2004). 
Tabla 1. El retorno social de las políticas culturales: perspectiva tradicional instrumental

\begin{tabular}{ll}
\hline Ámbito de repercusión & Algunos efectos de las políticas culturales \\
\hline Educación & Incremento de las competencias transferibles a la \\
& futura vida laboral y profesional. \\
& Aumento de la asistencia regular de los alumnos \\
y de la continuidad de los estudios. & Mejora de los vínculos entre la escuela y la comunidad. \\
& Mejora en la infraestructura y en los recursos de las \\
& escuelas. \\
\hline Incremento de las relaciones de las personas con el \\
entorno natural. \\
Conservación y preservación del patrimonio \\
arquitectónico y natural. \\
Promoción de productos y servicios ecológicamente \\
sostenibles. \\
Difusión del desarrollo medioambiental sostenible. \\
Creación de espacios de debate sobre los problemas \\
globales relativos al medio ambiente. \\
Mejora del entorno, el espacio y la difusión de la salud \\
pública. \\
Mejora de los tratamientos (el arte como instrumento \\
terapéutico). \\
Incremento de la actividad física y mental de los \\
pacientes. \\
\hline Salud
\end{tabular}

Fuente: elaboración propia.

ciente en la incorporación de la dimensión social en las políticas sanitarias. Esta mirada, que vincula la salud con el bienestar individual y social de las personas, parte de la idea de que las actividades artísticas y culturales (y el acceso y participación en ellas) pueden generar diferentes beneficios para los pacientes en particular, pero también para la ciudadanía en general.

Como síntesis de estas tres perspectivas, en la tabla 1 se presentan los ámbitos de repercusión de las políticas culturales y los efectos de la acción cultural de carácter público que esta perspectiva de análisis ha destacado.

\section{Límites de la perspectiva instrumental del retorno social de las políticas culturales}

Una de las características más relevantes de la perspectiva presentada es que se impulsa sobre todo desde experiencias e iniciativas públicas de gobiernos locales. Políticos y otros agentes implicados en la formulación de las políticas públicas en este nivel se interesan por las capacidades instrumentales del arte y la cultura. En este proceso, resulta crucial el hecho de que los profesionales de la cultura tengan, desde hace un tiempo, un nivel de visibilidad más alta en la arena política, pero, sobre todo, que esta visibilidad haya sido acompa- 
ñada de una capacidad creciente del sector para aprovechar y explotar recursos económicos asociados a presupuestos de otras políticas sectoriales (Belfiore, 2002 y 2006).

En definitiva, las transformaciones económicas, sociales y políticas de finales de los '70 y principios de los ' 80 son el contexto en el cual, ante el poco peso estructural de las políticas culturales (comparado con otras políticas públicas), sobre todo a nivel local, los agentes culturales reciben demandas para legitimar sus actuaciones con argumentos de tipo económico y social. Se trata de un fenómeno denominado policy attachment (Gray, 2007; Belfiore, 2006), es decir, una estrategia que permite a un sector de políticas públicas que tiene un peso en los presupuestos públicos relativamente bajo poder obtener recursos que le permitan conseguir sus objetivos.

Sin subestimar el impacto positivo de las iniciativas presentadas hasta aquí, y del conocimiento generado en las investigaciones científicas, resulta útil reflexionar sobre algunas de sus debilidades y también sobre las alternativas posibles. El proceso expansivo de la acción cultural, su vinculación directa con los objetivos de otros ámbitos y políticas públicas, puede derivar tanto en la sobrevaloración de su impacto real, como en la falta de profundidad conceptual en el análisis de sus resultados. El énfasis desmesurado y una dirección acrítica en la evaluación de las políticas públicas culturales resultan contraproducentes para su legitimidad y para su capacidad de incidencia real en la atención de las necesidades de la ciudadanía.

Esta mirada, basada en las externalidades generadas por las políticas culturales sin un necesario contrapunto crítico, ha generado expectativas y presiones desmesuradas sobre los agentes culturales. Así, se llega a asumir que la acción cultural financiada con recursos públicos debe concentrarse en atender prioridades de otras áreas de políticas y contribuir activamente a lograr sus objetivos (Belfiore, 2006). Eso comporta que, en lugar de debatir sobre qué y cómo hacen su tarea, las organizaciones e instituciones culturales necesitan demostrar de qué manera han contribuido a resolver las problemáticas instaladas en las agendas políticas más amplias, como, por ejemplo, la prevención del delito o el fracaso escolar.

Ante esta falta de reflexión y evaluación de las cualidades intrínsecas de la cultura, una perspectiva alternativa busca reconocer, más allá del impacto social de la cultura, su valor público (Holden, 2004). Se señala la necesidad de desarrollar un lenguaje que reconozca los elementos afectivos, intangibles, de la experiencia y la práctica cultural. Un hecho que no va en detrimento de la recolección de datos cuantitativos que miden el impacto de la cultura. Se trata de desarrollar políticas culturales que fomenten una cultura segura de su propio valor. Y poder impulsar una evaluación del valor público de las acciones de las organizaciones culturales, de los outcomes no necesariamente vinculados a categorías instrumentales. Se trata, en definitiva, de encontrar las vinculaciones de la cultura con valores permanentes como la equidad y la justicia.

La tabla 2 compara los elementos significativos de la primera mirada analizada, aquella que se ha ido consolidando desde la década de 1980 entre las 
Tabla 2. Las perspectivas sobre el retorno social de las políticas culturales

\begin{tabular}{lll}
\hline & Perspectiva tradicional & Nueva mirada \\
\hline Centrada en & Impacto social de la cultura. & Valor público de la cultura \\
\hline Identifica y destaca & $\begin{array}{l}\text { Externalidades: capacidades } \\
\text { instrumentales y estratégicas }\end{array}$ & $\begin{array}{l}\text { Transversalidades: beneficios } \\
\text { de las políticas culturales para }\end{array}$ \\
& de las políticas culturales. & la ciudadanía. \\
\hline
\end{tabular}

Fuente: elaboración propia.

iniciativas políticas y las investigaciones científicas, con los que presenta esta segunda lectura, en buena parte crítica con la perspectiva instrumental.

Por lo tanto, teniendo en cuenta que uno de los objetivos de este artículo es construir mejores criterios de evaluación de la acción pública en materia de cultura, en la sección siguiente se ha querido incorporar una mirada transversal que ayude a identificar aquellos elementos donde la acción cultural produce beneficios para la ciudadanía. Se trata de los efectos más significativos de las políticas culturales que pueden resultar útiles para construir una mirada transversal sobre el valor público de la cultura. El retorno social queda caracterizado, de esta manera, de forma equilibrada entre las capacidades instrumentales y estratégicas de la acción cultural y su valor público y a la vez intrínseco.

\section{Nuevas miradas sobre el retorno social de las políticas culturales}

¿Cómo construir entonces un concepto sólido, riguroso y profundo que dé cuenta de lo que significa el valor público de las políticas culturales, su retorno social? ¿Cómo conseguir que esa idea resulte de utilidad para el desarrollo de metodologías más completas para la evaluación de dichas políticas? ¿Cómo reconocer, al mismo tiempo, la importancia de la acción cultural para la calidad de vida de las personas y sus limitaciones? Con ánimo de abrir más que de concluir el debate, se presentan a continuación nueve ejes, ámbitos transversales en los cuales las políticas públicas culturales se entiende que generan repercusiones y beneficios a nivel social para el conjunto de la población. Reconocer las limitaciones en los resultados de la acción cultural pública no puede generar que la reflexión sobre sus beneficios se centre exclusivamente en aspectos instrumentales. Por eso, este artículo busca ampliar la mirada sobre qué es lo que debería analizarse y evaluar en la implementación de las políticas culturales. Consideramos que estos ejes pueden ser la base para el desarrollo de un sistema de indicadores para medir el retorno social de las políticas culturales.

1) Identidad y moral colectiva. Las políticas culturales contribuyen a la creación, a la potenciación y al mantenimiento de las identidades colectivas y del sentido de pertenencia comunitaria. Así, se desarrollan imaginarios y referentes comunes que se reflejan en la existencia de una ética compartida y en la protección de unos valores comunes aceptados. Con todas las precauciones y limitaciones al respecto, el retorno social de las políticas culturales puede faci- 
litar la inclusión de personas inmigradas en las diferentes comunidades de acogida. Las narraciones colectivas presentes en la comunidad (muchas veces vinculadas a un pasado común) pueden resultar lo suficientemente flexibles como para invitar a implicarse a quienes llegan, preservando la libertad del individuo para entrar y salir de los límites del colectivo.

Lógicamente, esta perspectiva presenta limitaciones. Una primera, de carácter metodológico, señalada en un estudio encargado por el Scottish Executive Education Department (Ruiz, 2004), da cuenta de la falta de evidencia sobre el impacto continuado a lo largo del tiempo de este tipo de políticas, que buscan la regeneración de los vínculos sociales y el fortalecimiento de las identidades compartidas. Una segunda, representativa de la dualidad que comporta la implementación de toda política cultural, se refiere a la herencia común (el pasado conservado y proyectado hacia el futuro) que puede promover la confianza y el sentido de pertenencia hacia una comunidad. Un estudio desarrollado por el Department of Canadian Heritage $(\mathrm{DCH})$ y el Canada Council for the Arts (Stanley, 2004) llama la atención sobre el carácter ficticio y de reconstrucción que comporta toda raíz y pasado presentado como común, fruto, en parte, de manipulaciones e interpretaciones de personas y acciones políticas, con el objetivo de modelar una identidad atractiva.

2) Capital social (I): cohesión e inclusión social. Las políticas culturales promueven la generación y la aceptación de normas y valores compartidos, que, a la vez, permiten la consolidación de los vínculos necesarios para el desarrollo de las comunidades. Estos procesos se traducen en una reducción significativa del aislamiento social y en una mejora de la cohesión social. Dentro el amplio abanico de significados que ha ido incorporando el concepto de capital social desde la formulación de James Coleman (1988) y Robert Putnam (2007), se destaca, en este caso, una de sus vertientes más importantes: la existencia de comunidades más cohesionadas, inclusivas y mejor capacitadas ${ }^{3}$.

Sin embargo, las políticas culturales que buscan de forma intencionada el desarrollo de normas comunes y redes sociales no siempre generan los efectos positivos de transformación que se esperan. Así, el análisis del programa $A r t$ and community development in Santa Ana, una iniciativa pública llevada a cabo en California en el año 2001, reveló la forma en que las divisiones y las desigualdades socioeconómicas de clase y etnia existentes entre la población se vieron agravadas de forma significativa (Barraket, 2005). Por lo tanto, más allá del contenido específico de los programas culturales y de si estos pretenden, de forma más o menos directa, un impacto de tipo social, la calidad de las políticas culturales y de su implementación continúa siendo un elemento clave para determinar su retorno social.

3. Para un análisis de experiencias de implementación de este tipo de políticas, puede consultarse el papel de la cultura para la cohesión social en políticas de la Comunidad Europea (Bina y Ijdens, 2007), las políticas culturales de carácter comunitarista en los Países Bajos (Etzioni, 1997 y 2004), o los efectos del arte (y sus limitaciones) en la inclusión social en el Reino Unido y Canadà (Barraket, 2005). 
3) Capital social (II): participación ciudadana y acción colectiva. Las políticas culturales fomentan el desarrollo de comunidades culturales (artistas, gestores, usuarios, público, etc.) con un alto grado de implicación ciudadana, lo que se traduce en el aumento de la participación y la acción colectiva. Entre las diferentes acepciones del concepto de capital social, se hace hincapié, en este caso, en cómo las políticas culturales contribuyen a la construcción de una ciudadanía activa y participativa a través de diferentes modalidades: el voluntariado y el asociacionismo en un primer orden, o la cooperación entre organizaciones y el trabajo en red en un segundo nivel. La pluralidad de factores que integran esta perspectiva de capital social queda reflejada en los diferentes tipos de análisis, heterogéneos pero no contradictorios. En este sentido, se pueden destacar dos ideas principales. Por un lado, las políticas culturales pueden generar más confianza en la actuación gubernamental y conseguir la implicación de la ciudadanía y la corresponsabilidad en la gestión pública (Cox, 1995). Por otra parte, las políticas culturales evidencian la necesidad de las sociedades de actuar de forma colectiva. Así, la participación ciudadana se entiende como motor de la democracia (Putnam, 2001).

4) Reconocimiento y gestión del conflicto. Las políticas culturales pueden fomentar una idea de democracia más abierta y accesible que permite afrontar los conflictos sociales dentro de un marco simbólico. Se reconoce el conflicto como motor social y la política, como espacio para su gestión. Las concepciones sobre qué se entiende por grupo y comunidad cultural (y sus límites) se vuelven más flexibles y amplias. En este sentido, el análisis de las políticas culturales de promoción de la diversidad cultural y de la interculturalidad resulta de gran utilidad. Las dificultades detectadas en la implementación de determinadas políticas basadas en conceptos tradicionales como el multiculturalismo han llevado, en algunos casos, a la revisión de este tipo de acción pública. Son pocos pero significativos los intentos por adoptar una noción realista de las potencialidades del arte y la cultura.

Así, las investigaciones sobre hibridación cultural han significado un cambio de perspectiva sobre el multiculturalismo, ya que desarrollan una alternativa que reconoce que la fusión y el sincretismo entre culturas pueden generar contradicciones. Se identifica como elemento dinamizador de las políticas culturales la existencia de conflictos generados a partir de los recientes procesos de interculturalidad, pero, a la vez, este reconocimiento ha permitido que determinadas políticas culturales abandonen un tipo de discurso y acción de carácter esencialista sobre lo que representa la identidad. El arte y la cultura, en estos casos, dejan de percibirse como recursos casi mágicos para la reconciliación planetaria, la integración y la cohesión universal (Bianchini, 1995; Bianchini y Bloomfield, 2004; Garcia Canclini, 2001).

5) Revalorización de las denominadas «clases no productivas». Se trata de grupos que acostumbran a estar infrarrepresentados entre las audiencias culturales, y sus niveles de participación y consumo cultural también resultan escasos. El colectivo de los adultos mayores es el más importante entre aquellos conside- 
rados, desde el paradigma industrialista, como clases no productivas, un segmento que también incluye personas en riesgo de exclusión social, con problemas de movilidad, minorías étnicas o incluso algunas comunidades locales. Las políticas culturales contribuyen al desarrollo de personas con intereses, recursos y capital cultural propio. Por lo tanto, esto hace que resulten, sobre todo en el momento de llegar a las últimas etapas de la vida, más independientes. El retorno social de las políticas culturales implica la mejora de la percepción que los grupos señalados tienen de ellos mismos, los acerca a los otros individuos y los incluye en las comunidades de pertenencia.

6) Desarrollo autónomo y promoción de la creatividad de las personas. El retorno social de las políticas culturales está directamente vinculado al desarrollo autónomo de los individuos y a la promoción de su creatividad para interpretar, entender y hacer entender a los demás el mundo que los rodea. Implica la formación integral de personas capacitadas para responder a un mundo en constante transformación a través de procesos de innovación social y económica.

La aparición de nuevos modelos de trabajo en red señalan que no sólo instituciones formalizadas, como las universidades o los institutos de investigación, están preparadas para producir conocimiento, sino que otras organizaciones sociales y culturales también desarrollan procesos de innovación (Yproductions, 2008). Este tipo de procesos resulta clave en un momento en que la formación integral de las personas adquiere, cada vez más, mayor centralidad en las políticas públicas. En ocasiones, esta perspectiva comporta la revalorización de la subjetividad de las personas como elemento central de la creatividad. Se desarrollan mecanismos que permiten captar sus capacidades para desarrollar e implementar nuevas ideas (productos, servicios y modelos) que buscan suplir carencias sociales. Ante los modelos externos que impulsan la instrumentalización de la cultura, las políticas culturales pueden promover, a través de la innovación social, la instrumentalización de la economía.

7) Nuevas centralidades. Las políticas culturales contribuyen a la generación y al reconocimiento de nuevas centralidades, más allá del entorno urbano. En muchas ocasiones, estos procesos implican la revitalización de las economías de las comunidades locales. Las ciudades son el lugar donde se desarrollan gran parte de las acciones y las actividades sociales y donde se generan conocimientos útiles para la evolución de las sociedades. El espacio urbano, por definición, cuenta con la infraestructura suficiente para que las personas desarrollen todas las actividades económicas, sociales y culturales necesarias en este sentido. Actualmente, sin embargo, el crecimiento indiscriminado de las ciudades y la complejidad en su gestión provoca que se busquen nuevas alternativas a estas centralidades. Es en este sentido que se identifican dos tipos de retorno social de las políticas culturales. Por un lado, las políticas culturales permiten que se generen y se reconozcan nuevas centralidades vinculadas al desarrollo de las comunidades locales, que funcionan o son interpretadas como nuevos yacimientos de conocimientos. Y, por otra parte, el retorno social de las políticas 
culturales se traduce en la revitalización de las economías de las comunidades locales y la regeneración de vínculos sociales en las zonas más desfavorecidas.

Uno de los ejemplos analizados en este sentido es el del rol de las políticas culturales en los programas de desarrollo regional en Australia (Mills y Brown, 2004). El programa impulsó, a partir del año 2002, la reforma de determinadas instituciones político-económicas, adoptando una perspectiva de base cultural, otorgando un caràcter central a valores y conocimientos de las comunidades y priorizando la aportación de las artes y las iniciativas culturales de base tradicional local. Entre los resultados, se obtuvo una mejora en la viabilidad económica, y al mismo tiempo se reforzó la cohesión social e impulsó su autonomía de la gestión de los recursos naturales.

8) Reinterpretación del paisaje. Las políticas culturales generan un cambio significativo de actitud en la reinterpretación del paisaje, que se entiende como construcción cultural y, por lo tanto, colectiva. De esta manera, aumenta la conciencia colectiva y la preocupación por la sostenibilidad. Las transformaciones que el paisaje experimenta constantemente, debido a la expansión del fenómeno urbano en el territorio y al desarrollo de la capacidad tecnológica de transformación de la naturaleza, cada vez adoptan ritmos más acelerados. Frente a la dinámica tradicional de desarrollo industrial, en el siglo XXI, las propuestas de ordenación territorial van más encaminadas a la combinación del binomio formado por cultura y naturaleza. En este contexto, las políticas culturales pueden resultar fundamentales para contribuir a desarrollar en los individuos una perspectiva más amplia de la sostenibilidad del paisaje e impulsar la creatividad, la imaginación y la participación en su gestión y transformación (Haley, 2008).

9) Transformación del espacio urbano. Se trata de uno de los aspectos más controvertidos, complejos y, por lo tanto, más significativos del retorno social de las políticas culturales, que merece una atención especial. Diferentes estudios han identificado las evidencias positivas y negativas del impacto de los procesos de regeneración urbana, donde la cultura (y sus políticas públicas) ha adquirido un papel nuclear ${ }^{4}$. Actualmente, una de las miradas sobre la evolución del espacio urbano más discutidas y, a la vez, más adoptadas por los gobiernos locales es la de la teoría de las clases y las ciudades creativas (Florida, 2002; Knudsen et al., 2007). Para esta perspectiva, las ciudades con una alta densidad de instituciones, organizaciones y actividades artísticas y culturales están en mejores condiciones para afrontar los retos del cambio en los modelos de desa-

4. Entre los impactos positivos, se han destacado la recuperación y la reutilización de edificios y espacios públicos, lo que ha generado como retorno la disminución de áreas consideradas marginales y de acceso prohibido, de la polarización socioeconómica, de los niveles de contaminación, de las tasas de delitos, etc. (Bianchini: 1995; Bianchini y Bloomfield, 2004; Landry, 2004). Entre los negativos, se han analizado los efectos del modelo «ciudad 24 horas", así como los conflictos de intereses entre residentes y comerciantes, la exclusión de muchos colectivos sociales de la programación cultural o el incremento desmedido del valor de la propiedad y el desplazamiento de los residentes habituales y comerciantes de las áreas intervenidas (gentrificación). 
rrollo económico. La regeneración laboral y el crecimiento económico son parte de los efectos que pueden tener el desarrollo de una política cultural que apoye a este modelo. Ahora bien, se trata de un modelo discutido, porque se considera que implica a la ciudadanía en proyectos de regeneración superficiales y procesos de gentrificación en ciudades que presentan grandes niveles de desigualdades socioeconómicas. Se critica, así, lo que se considera una dinámica de mercantilización (commodification) de los recursos culturales y de la misma idea de tolerancia (Peck, 2005).

Uno de los ejemplos de políticas culturales que podemos mencionar y analizar es el conjunto de decisiones políticas vinculadas a la construcción de equipamientos culturales en el centro histórico de la ciudad de Viena (Museumsquartier Wien) (De Frantz, 2005). La aparición de conflictos simbólicos en relación con el papel de la cultura en la ciudad provocaron que un proyecto de regeneración urbana incorporara un proceso deliberativo de transformación del espacio público, con un papel destacado de los propios residentes, donde la conservación de la identidad histórica se resignifica como crítica a ciertos aspectos de la modernidad.

\section{Nuevas perspectivas, ¿nuevos indicadores?}

Identificada la complejidad que comporta una mejora en la comprensión del retorno social de las políticas culturales, de la magnitud de los beneficios que generan, pero también de sus limitaciones, el reto es poder trasladar esa mirada integral al desarrollo específico de indicadores útiles para su evaluación.

¿Cuál es el estado actual, a nivel internacional, del desarrollo de indicadores culturales? Desde principios de la década de 1970, buena parte de los esfuerzos en la investigación sobre políticas culturales se ha concentrado en la elaboración de indicadores. No obstante, las experiencias llevadas a cabo permitieron comprobar las limitaciones y las dificultades en la aplicación uniforme de los indicadores para diferentes tipos de políticas culturales y en diferentes territorios (Gouiedo, 1993). Es por eso que, por ejemplo, el Instituto de Estadísticas de la UNESCO, que, desde los años setenta, elaboraba indicadores comunes para la mayoría de políticas culturales, con el tiempo decidió abandonar esta recogida sistemática y universal de información (Bonet, 2004).

Sin embargo, como se ha avanzado, sí que existe un cierto acuerdo en la investigación científica internacional (sobre todo, nuevamente, la anglosajona) sobre la necesidad de continuar impulsando un cambio en el modelo de evaluación de políticas culturales. Un paso desde una perspectiva centrada en la consideración de valores estéticos e instrumentales de las artes y la cultura hacia una mirada realista, pero al mismo tiempo más compleja. Esta mirada afronta el reto de medir el retorno social generado, sin ignorar el impacto económico que toda actividad cultural comporta. Lógicamente, esta tendencia implicará la adopción de conceptos con límites poco definidos y fácilmente manipulables (diversidad cultural, inclusión social, etc.), situación sobre la que hemos advertido ya en este artículo. 
Tabla 3. Clasificación de los indicadores culturales

\begin{tabular}{|c|c|}
\hline Tipo de indicadores & Tipos de ejemplos utilizados \\
\hline $\begin{array}{l}\text { 1. Sobre la mejora del medio } \\
\text { ambiente y la regeneración } \\
\text { de espacios públicos }\end{array}$ & $\begin{array}{l}\text { Obras y espacios arquitectónicos declarados } \\
\text { de interés patrimonial. } \\
\text { Consideración y función del patrimonio entre } \\
\text { los objetivos de los planes urbanísticos. }\end{array}$ \\
\hline $\begin{array}{l}\text { 2. Sobre el bienestar individual } \\
\text { y desarrollo personal }\end{array}$ & $\begin{array}{l}\text { Voluntarios que participan en actividades culturales } \\
\text { y cantidad de horas dedicadas } \\
\text { Personas que perciben la participación en actividades } \\
\text { culturales como beneficio personal. }\end{array}$ \\
\hline 3. Del desarrollo económico & $\begin{array}{l}\text { Volumen de exportación de productos artísticos. } \\
\text { Atractivo de las ciudades para los trabajadores } \\
\text { calificados. }\end{array}$ \\
\hline $\begin{array}{l}\text { 4. Del capital social y del } \\
\text { desarrollo comunitario }\end{array}$ & $\begin{array}{l}\text { Actividades culturales organizadas por comunidades } \\
\text { étnicas y minoritarias. }\end{array}$ \\
\hline $\begin{array}{l}\text { 5. De la vitalidad cultural del } \\
\text { entorno }\end{array}$ & $\begin{array}{l}\text { Equipamientos culturales por habitante. } \\
\text { Densidad de organizaciones del tercer sector } \\
\text { cultural. }\end{array}$ \\
\hline $\begin{array}{l}\text { 6. De la envergadura y la } \\
\text { estructuración del sector } \\
\text { cultural }\end{array}$ & $\begin{array}{l}\text { Número de empresas del sector cultural. } \\
\text { Inclusión de las industrias culturales en las } \\
\text { estrategias globales de desarrollo económico. }\end{array}$ \\
\hline
\end{tabular}

Fuente: elaboración propia a partir de Madden (2005), Simons y Dang (2006), Mercer (2003) e International Intelligence on Culture (2005).

En definitiva, si la evaluación del retorno social de las políticas culturales no es ni mucho menos una tarea sistematizada en el ámbito internacional, sí que existe algún esfuerzo aislado por clasificar los diversos indicadores culturales (en general y ya no exclusivamente del impacto social de la cultura) que actualmente se utilizan con mayor frecuencia. Así, se avanza en una tarea más que recomendable, como la de discriminar entre indicadores generalistas (que intentan medir el impacto de la cultura en el total de la población) de los que se centran en determinadas prácticas culturales y sus efectos, en algunos colectivos en concreto y en escalas territoriales diferenciadas (niveles nacionales, regionales, locales, etc.). El trabajo de Simons y Dang (2006) es un ejemplo en este sentido, que además diferencia pero entiende como complementarios los indicadores de tipo cuantitativo y cualitativo. En la tabla 3, se presentan las seis categorías de indicadores culturales que distinguen diferentes autores, así como algunos de los ejemplos más utilizados en las diversas investigaciones a nivel internacional.

\section{Nuevos indicadores, ¿antiguos problemas?}

Como se observa en la tabla 3, el tipo de indicadores culturales utilizados es realmente muy diverso, y no solamente porque pueden incorporar informa- 
ción de tipo cuantitativo o cualitativo, sino también porque combinan valoraciones económicas, estéticas y políticas con percepciones sobre los beneficios personales de la cultura. Esta compleja perspectiva descarta, así, que cualquier tipo de impacto de las políticas culturales sea susceptible de ser medido de forma directa y homogénea en diferentes escalas comunitarias y territoriales. Por lo tanto, miradas de tipo universalista, como, por ejemplo, el modelo de evaluación del impacto social de la participación en actividades artísticas y culturales de Matarasso (1997), han sido criticadas por su falta de validez interna y por la ambigüedad conceptual de partida y en el momento de analizar los resultados obtenidos (Belfiore, 2006).

La incorporación, por parte de muchas administraciones públicas, de metodologías de evaluación de este tipo ha impulsado la utilización directa de estadísticas descontextualizadas como si se tratara de indicadores. Por ejemplo, se ha confundido una estadística estimada del número de personas que trabajan en el sector cultural con un indicador del impacto social de la cultura en sí mismo, sin especificar ningún tipo de medida relativa. Este tipo de confusión implica que los datos recogidos no permiten identificar los fenómenos que se buscan explicar, con lo cual se establecen relaciones de causa-efecto equivocadas o se transforman situaciones anecdóticas en afirmaciones contundentes pero poco rigurosas.

El esfuerzo por identificar indicadores clave se explica por la necesidad de los poderes públicos de contar con herramientas útiles para alcanzar mayores niveles de eficiencia y equidad en la asignación de recursos. Pero el desarrollo de indicadores para el sector cultural debería tener en cuenta los peligros que ha supuesto y comporta su uso en la evaluación de políticas públicas. Autores como Peter Smith (1995) han advertido de algunas de les tendencias más nocivas en este sentido. Los problemas disfuncionales y los fenómenos surgidos como consecuencia de una mala gestión de los indicadores y la información son diversos. En la tabla 4, se presentan algunos de estos fenómenos, sus características principales y ejemplos de indicadores relacionados con estos inconvenientes.

En definitiva, esta explicación apunta a especificar la dificultad y las limitaciones que conlleva la construcción de indicadores válidos y contrastados para el análisis de las políticas culturales. Si se busca evitar el bosque de indicadores que lleven a la pérdida de capacidad para señalar y destacar fenómenos particulares, será necesario reconocer que una de las limitaciones principales es la imposibilidad de desarrollar un modelo común y estandarizado, que pase por alto las particularidades territoriales y las de los diferentes ámbitos y sectores de política cultural. Así, en la evaluación de políticas culturales, debería ser posible construir y combinar indicadores que recojan información cuantitativa y cualititativa. Por ejemplo, que den pauta de las tendencias de consumo cultural, al mismo tiempo que de las percepciones y las actitudes de la ciudadanía sobre los efectos del arte y la cultura.

Construir indicadores para evaluar políticas culturales no es una tarea meramente técnica. Los indicadores deberían poder reflejar una perspectiva com- 
Tabla 4. Tendencias nocivas en la utilización de indicadores en la evaluación de políticas públicas

\begin{tabular}{|c|c|}
\hline Fenómenos & Características \\
\hline Visión de túnel & $\begin{array}{l}\text { Énfasis desproporcionado en la evaluación de los objetivos } \\
\text { a través de los efectos cuantificables, en detrimento de la } \\
\text { medición de aspectos que no pueden cuantificarse. } \\
\text { Ejemplo: utilización de estadísticas descontextualizadas } \\
\text { como indicadores culturales. }\end{array}$ \\
\hline Suboptimización & $\begin{array}{l}\text { Confusión entre los objetivos generales de una } \\
\text { organización y los objetivos individuales de sus } \\
\text { trabajadores. } \\
\text { Ejemplo: indicadores que miden el número de expedientes } \\
\text { resueltos por cada trabajador sobre el total. }\end{array}$ \\
\hline Miopía & $\begin{array}{l}\text { Tendencia a evaluar los efectos de una política a corto } \\
\text { plazo, cuando, en realidad, sus resultados sólo pueden } \\
\text { ser contemplados en un período amplio. } \\
\text { Ejemplo: indicadores que evalúan las políticas de } \\
\text { formación a través de la inserción laboral en un período } \\
\text { de seis meses. }\end{array}$ \\
\hline Fijación en la medida & $\begin{array}{l}\text { Dificultad para desarrollar indicadores capaces de evaluar } \\
\text { los objetivos de las políticas en su complejidad. Así, se } \\
\text { prefiere adaptar los instrumentos de intervención } \\
\text { política y dejar de lado la evaluación de los objetivos. } \\
\text { Ejemplo: indicadores que toman como éxito de la política } \\
\text { determinados resultados electorales. }\end{array}$ \\
\hline Falsa representación & $\begin{array}{l}\text { Indicadores que pueden comportar incentivos para } \\
\text { manipular la información, de manera que se pueda } \\
\text { demostrar el éxito de las políticas adoptadas. } \\
\text { Ejemplo: indicadores que miden el número de expedientes } \\
\text { resueltos por cada trabajador sobre el total. }\end{array}$ \\
\hline Falsa interpretación & $\begin{array}{l}\text { Utilización de indicadores de tipo racional instrumental } \\
\text { que comportan patrones de interpretación y significados } \\
\text { que se desvían de los objetivos políticos. } \\
\text { Ejemplo: indicadores que clasifican las organizaciones } \\
\text { educativas y culturales en formato de tablas de } \\
\text { resultados y competición. }\end{array}$ \\
\hline Manipulación & $\begin{array}{l}\text { Manipulación del comportamiento de los actores } \\
\text { evaluados a partir de las características de los } \\
\text { indicadores. } \\
\text { Ejemplo: indicadores que miden el aumento de } \\
\text { productividad de los trabajadores comparándolo } \\
\text { con el período inmediatamente anterior. }\end{array}$ \\
\hline Osificación & $\begin{array}{l}\text { Rigidez en el sistema de evaluación que comporta } \\
\text { la parálisis de las organizaciones. }\end{array}$ \\
\hline
\end{tabular}

Fuente: elaboración propia a partir de Smith (1995). 
pleja y suficientemente amplia del fenómeno social que se busca medir, considerando particularidades territoriales y motivaciones de los actores implicados. Incluso una prueba de la envergadura y la calidad de un indicador es su capacidad para dar cuenta de diferentes aspectos de la realidad social a partir de una misma medida. Para conseguir este nivel de amplitud, resulta útil que el ejercicio de construcción de indicadores pueda implicar, de alguna manera, a los agentes que probablemente serán los futuros evaluados. Y eso incluye, para el caso del retorno social de la cultura, los miembros de los diferentes subsectores de ese ámbito, pero, fundamentalmente, representantes y responsables de otras políticas públicas sociales.

\section{Una propuesta de doce índices del retorno social de las políticas culturales}

Hasta aquí, hemos intentado dar cuenta de la complejidad conceptual que implica evaluar el retorno social de las políticas culturales, sin dejar de señalar la necesidad de construir una mirada integral y realista sobre sus beneficios para la ciudadanía. También, hemos insistido en el cambio de modelo de evaluación de las políticas culturales en general (y de su retorno social en particular) y en la dificultad para desarrollar indicadores coherentes y científicamente válidos. A continuación, se presentan doce índices que, tras el análisis documental realizado, consideramos que resultan significativos y adecuados por su calidad y su capacidad para dar cuenta del retorno social de las políticas culturales. Se trata de doce magnitudes, medidas que pretenden ser un primer paso útil para desarrollar, teniendo en cuenta las precauciones indicadas, un sistema de indicadores realista y ajustado al contexto territorial y a los actores implicados según el caso.

1. Indice sobre el grado de diversidad cultural, social y económica de la audiencia de las actividades culturales. Busca medir el retorno social que generan las políticas culturales en relación con la creación, la potenciación y el mantenimiento de las identidades colectivas y del sentido de pertenencia comunitaria, así como con la recreación de vínculos sociales en espacios compartidos.

2. Indice sobre el grado de asistencia y participación en grupo en actividades culturales. Conocer con quién y hasta qué punto las personas participan en grupo en la oferta cultural puede resultar de utilidad para identificar si realmente el retorno social de las políticas culturales se traduce en una reducción del aislamiento social o la resocialización de las personas, con lo cual aumenta el capital social y la cohesión en este sentido.

3. Indice de conocimiento de lenguas y de lectura de la gente adulta. Recuperando el debate sobre las denominadas "clases no productivas», este índice puede permitir establecer criterios iniciales para evaluar cuál es el grado de independencia de la gente adulta y cómo las políticas culturales comportan el desarrollo de personas con intereses, recursos y capital cultural propio.

4. Indice de porcentaje de personas que perciben la cultura como beneficio personal. Se trata de una medida que no busca identificar una evidencia material 
concreta, sino más bien la percepción de las personas sobre el retorno social de las políticas culturales. Es decir, si se perciben los beneficios de la acción cultural, no tanto en clave de outcome material directo, sino respecto a la autonomía y la promoción de la creatividad de las personas, sus capacidades para interpretar y responder al contexto de transformación social.

5. Indice de presencia de la innovación social en las organizaciones culturales públicas y privadas. Se busca, así, medir la manera como las políticas culturales han incorporado, entre sus objetivos, la innovación no sólo en términos económicos, sino también en cuanto a las capacidades de dar respuestas a nuevas necesidades derivadas del cambio de contexto social, así como comprobar en qué grado se produce conocimiento, no sólo desde instituciones especializadas, sino también desde otro tipo de organizaciones culturales.

6. Indice de distribución de la densidad de actividades culturales e instituciones entre los centros y las periferias (Mills y Brown, 2004). Este índice pretende identificar si se han desarrollado procesos democráticos e inclusivos de descentralización de las políticas culturales. También, de qué manera el retorno social se traduce en la generación y el reconocimiento de nuevas centralidades, más allá del espacio urbano, a través del desarrollo de comunidades locales que funcionan o son interpretadas como nuevos yacimientos de conocimientos.

7. Indice sobre el incremento del turismo vinculado a proyectos de revitalización de las tradiciones y el patrimonio natural y cultural de las comunidades locales (Galvani, 2001). Como el índice presentado anteriormente, se pretende identificar si el retorno social de las políticas culturales se manifiesta en el desarrollo de nuevas centralidades más allá de los centros urbanos y en la revitalización de las comunidades locales.

8. Indice sobre el grado de participación de la población local joven en las actividades y los programas culturales. De forma complementaria a los dos índices anteriores, se busca identificar cuál es el grado de revitalización de las comunidades locales desde una perspectiva más integral y compleja. Por eso, se toma como punto de referencia la participación de la población más joven en la oferta cultural descentralizada.

9. Indice sobre el grado de presencia en las ciudades de cientificos e ingenieros, bohemios (artistas, músicos, escritores, etc.) y gays, combinado con el indice de inversión en $I+D$ (Florida, 2005). Este índice, elaborado por Richard Florida e implementado en forma de estrategias políticas por muchos gobiernos locales, busca demostrar el nivel de desarrollo de las llamadas "ciudades y clases creativas», así como la influencia que pueden ejercer las políticas culturales en este tipo de procesos. Siendo conscientes de las implicaciones, las críticas y las limitaciones de una medida como esta, consideramos que puede resultar de utilidad si se vincula directamente con el índice número 10 presentado a continuación.

10. Indice sobre la evolución del valor de la propiedad y del perfil socioeconómico de los residentes y los comerciantes de los territorios donde se han implementado estrategias de regeneración urbana (Simons y Dang, 2006). Este 
índice mide las implicaciones que pueden tener los procesos de regeneración urbana donde las políticas culturales tienen un papel significativo. Además, complementa la mirada sobre el impacto del desarrollo de las llamadas «ciudades creativas».

11. Indice sobre los lugares declarados como patrimonio cultural y natural que, además, quedan reconocidos como recursos en los planes de desarrollo territorial. Este índice, incluido en los documentos de la organización no gubernamental Consejo Internacional de Monumentos y Espacios, resulta de utilidad para identificar cuál es el grado de reconocimiento del papel del patrimonio cultural y natural por parte de las instituciones públicas.

12. Indice sobre el número de espacios naturales declarados paisaje cultural y sobre su presencia en los programas de actividades de las organizaciones culturales (Haley, 2008). Índice que busca comprobar si las políticas culturales pueden resultar significativas para contribuir a desarrollar en los individuos una perspectiva más amplia de la sostenibilidad del paisaje, impulsar la creatividad, la imaginación y la participación en su gestión y transformación.

\section{Consideraciones finales}

Para concluir, pretendemos llamar la atención sobre algunas de las aportaciones más significativas de este artículo. También, nos interesa reflexionar sobre la utilidad que puede tener para la Administración pública (y para el resto de los actores implicados) apostar por un cambio en los modelos de evaluación de las políticas culturales.

En su breve historia de vida, las políticas culturales han ido acumulando objetivos e implementando nuevos instrumentos de intervención. La idea de cultura desplegada en la acción pública se ha expandido hasta el punto que, actualmente, la necesidad de las políticas culturales se justifica en buena parte por sus externalidades, por la contribución de la cultura a la consecución de los objetivos de otras agendas políticas. La evaluación de políticas culturales o bien continúa basada en antiguos criterios (de orden estético y centrada en las estadísticas de consumo cultural) o bien adquiere una dirección acrítica, que obliga a las instituciones culturales a intentar demostrar de qué manera han ayudado a resolver problemáticas como la prevención del delito o el fracaso escolar. Este proceso, globalmente, ha resultado contraproducente para la legitimidad de las políticas culturales y su capacidad de incidencia real en la atención de las necesidades de la ciudadanía y la generación de beneficios para su conjunto.

El reto se encuentra, entonces, en desarrollar una política cultural (y un modelo para su evaluación) basada en el valor público de la cultura, que busca el reconocimiento de los elementos afectivos, intangibles, de la experiencia y la práctica cultural, así como de su vinculación con valores permanentes. Reconocer las limitaciones en los resultados de la acción cultural pública no puede derivar en una reflexión sobre sus beneficios centrada con exclusividad en aspectos instrumentales. Asumiendo las dificultades metodológicas, es fun- 
damental comprender de qué manera las políticas culturales generan un retorno social para la ciudadanía: el desarrollo de identidades colectivas flexibles, la construcción de valores compartidos, la regeneración de vínculos sociales, la acción política democrática, el reconocimiento del conflicto en un marco simbólico, el desarrollo personal autónomo y creativo, la democratización en la generación y el acceso al conocimiento, el gobierno plural e inclusivo del territorio, etc. ${ }^{5}$. De esta manera, el retorno social de las políticas culturales quedaría caracterizado de forma equilibrada entre las capacidades instrumentales y estratégicas de la acción cultural y su valor público y a la vez intrínseco. Por lo tanto, más allá del contenido específico de los programas culturales y la búsqueda más o menos directa de su impacto social, la calidad de las políticas culturales y de su implementación continúa siendo un elemento clave para determinar su retorno social. Conviene advertir que, si no se tiene en cuenta la necesidad de una consistencia interna relativa entre valores o elementos conceptuales de las políticas culturales y sus aspectos sustantivos (ámbitos en que se despliegan) y sus aspectos operativos (formas concretas en que se implementan), las debilidades y las contradicciones harán peligrar los resultados esperados.

Esta perspectiva no sólo asume el reto de trabajar con conceptos que tienen límites poco definidos y que se prestan a la manipulación, sino también de trasladarlos al proceso de elaboración de modelos de evaluación de políticas culturales, con sus correspondientes sistemas de indicadores. Nuevamente, se trata de una tarea necesaria, que evita la utilización directa, por parte de un buen número de administraciones e investigaciones científicas, de estadísticas descontextualizadas como si se tratara de indicadores, sobre todo cuando, en la actualidad, el tipo de indicadores culturales utilizados, tanto por las investigaciones básicas como los casos aplicados por las instituciones públicas, es realmente muy diverso. Por eso, este artículo presenta doce índices que consideramos adecuados, más que para establecer una base normativa sobre los indicadores del retorno social de las políticas culturales, para contribuir al debate y a la generación de conocimiento sobre el valor de la cultura y las posibilidades de evaluación realista, contextualizada, compleja y rigurosa de sus políticas. No podemos dejar de pensar que se trata de un debate del todo útil para el diseño de metodologías que permitan obtener mejor información sobre la eficacia de les políticas culturales, la transparencia en su desarrollo y las consecuencias de su implementación.

5. Si bien no contamos con el espacio adecuado para poder analizar en profundidad las relaciones entre políticas culturales y políticas de comunicación, cabe decir que se trata de una perspectiva necesaria y relevante. Como explica Barbero (2008), las políticas culturales parecen construirse hoy sin pensar en los procesos de comunicación entre las culturas. La evaluación del retorno social de las políticas culturales no puede dejar de preguntarse de qué manera éstas cuentan con las transformaciones que se están operando en las propias sociedades, en particular, la creación, la difusión y el acceso a la cultura a través de Internet. 


\section{Referencias bibliográficas}

BARBERO, Jesús Martín (2008). «Políticas de la comunicación y la cultura: Claves de la investigación». Documentos CIDOB Dinámicas interculturales, 11.

BARRAKET, Jo (2005). "Putting people in the picture? The role of the arts in social inclusion». Social Policy Working Paper, 4.

BELFIORE, Eleonora (2002). "Art as a means of alleviating social exclusion: Does it really work? A critique of instrumental cultural policies and social impact studies in the UK». International Journal of Cultural Policy, 8 (1), 91-106.

- (2006). "The social impacts of the arts - myth or reality?». En: MirzA, Munira (ed.) Culture Vultures: Is UK arts policy damaging the arts? Londres: Policy Exchange.

BiANCHINI, Franco (1993). "Remaking European cities: the role of cultural policies». En: BiAnCHINI, Franco y PARKINSON, Michael (ed.). Cultural policy and urban regeneration: The West European experience. Manchester: Manchester University Press.

- (1995). «Cultural considerations in inner city regeneration». En: Culture and neighbourhoods. Strasbourg: Council of Europe Publishing.

Bianchini, Franco y BlOOMfield, Jude (2004). Planning for the intercultural city. Stroud: Comedia.

BINA, Vladmír y IJDENS, Teunis (2007). Social participation and cultural policy: a position paper. Den Haag: Ministerie van Onderwijs, Cultuur en Wetenschap.

Bloomfield, Jude (1993). «Bologna: a laboratory for cultural enterprise». En: BiAnCHINI, Franco y PARKinson, Michael (eds.), Cultural policy and urban regeneration: The West European experience. Manchester: Manchester University Press.

BONET, Lluís (2004). «Reflexiones a propósito de indicadores y estadísticas culturales». Boletín GC. Portal Iberoamericano de Gestión Cultural.

[www.gestioncultural.org/boletin/pdf/Indicadores/LBonet-Indicadores.pdf , consulta: 16 de abril de 2009]

CAVE, Ben y Couts, Adam (2002). Health Evidence Base for the Mayor's Draft Cultural Strategy. Londres: South East London Strategic Health Authority/East London and the City Health Action Zone.

Coleman, James S. (1988). "Social Capital in the Creation of Human Capital». American Journal of Sociology, 94, 95-120.

Cox, Eva (1995). A Truly Civil Society. Sidney: Boyer Lectures, ABC Books.

DE FrantZ, Monika (2005) «From Cultural Regeneration to Discursive Governance: Constructing the Flagship of the 'Museumsquartier Vienna' as a Plural Symbol of Change». International Journal of Urban and Regional Research. 29 (1), 50-66.

Duxbury, Nancy; GilletTe, Eileen y PePPER, Kaija (2007). Exploring the cultural dimensions of sustainability. Vancouver: Creative City Network of Canada. [http://www.creativecity.ca/news/special-edition-4/index.html, consulta: 10 de abril de 2009]

EtzIOnI, Amitai (1997). The New Golden Rule: Community and Morality in a Democratic Society. Nueva York: Basic Books.

- (2004). The Common Good. Cambridge: Polity Press.

FlORIDA, Richard (2002). The Rise of the Creative Class. Nueva York: Basic Books.

- (2005). The Flight of the Creative Class. Nueva York: HarperCollins.

Galvani, Marina (2001). The Social Impact of Culture - Empirical Content. Thinking outside the Box: Arguments for IADB's Involvement in the Preservation and Develipment of Heritage. Washington, D.C.: Inter-American Development Bank. 
SCOTTISH ARTS CounCIL (2008). The evidence base for arts and culture policy. A brief review of selected literature. Edinburgh: Scottish Arts Council...

García Canclini, Néstor (2001), Culturas híbridas. Buenos Aires: Paidós.

GouiEDO, Leif H. (1993). «Proposals for a set of cultural indicators». Statistical Journal of the United Nations, ECE, 10 (3).

GraY, Clive (2002). «Local Government and the Arts». Local Government Studies, 28 (1), 77-90.

- (2007). "Commodification and instrumentality in cultural policy». International Journal of Cultural Policy 13 (2), 203 - 215.

Haley, David (2008). "Arts in ecology: Questions of foresight». Music and Arts in Action, 1 (1), 21-35.

Hawkes, Jon (2001), The Fourth Pillar of Sustainability: Culture's essential role in public planning. Victoria: Common Ground Publishing and Community Cultural Development Network.

Holden, John (2004). Capturing cultural value. Londres: DEMOS.

International Intelligence on Culture (2005). Hong Kong Arts \& Cultural Indicators. Hong Kong Arts Development Council.

JEANNOTTE, M. Sharon (2008). "Shared spaces: Social and economic returns on investment in cultural infrastructure". En: DUXBURY, Nancy. Under Construction: The State of Cultural Infrastructure in Canada. Vancouver: Simon Fraser University.

Knudsen, Brian; FloridA, Richard; Gates, Gary y STOLARICK, Kevin (2007). «Urban density, creativity, and innovation». Creative Class Group Working Paper.

LANDRY, Charles (2004). Culture \& Regeneration: an evaluation of the Evidence. Nottingham: Comedia.

Madden, Christopher (2005). «Statistical Indicators for Arts Policy». Cultural Trends, $14(3), 55$.

MATARASSO, François (1997). Use or Ornament? The Social Impact of Participation in the Arts. Londres: Comedia.

MERCER, Colin (2003). Passer des indicateurs à la governance au courant dominant du grand public: Outils de politiques culturelles et de citoyenneté. Nottingham.

Miller, Toby y Yúdice George (2004). Política cultural. Barcelona: Gedisa.

Mills, Deborah y Brown, Paul (2004). «Art and Wellbeing: A guide to the connections between Community Cultural Development and Health, Ecologically Sustainable Development, Public Housing and Place, Rural Revitalisation, Community Strengthening, Active Citizenship, Social Inclusion and Cultural Diversity». Australia Council for the Arts.

Morris, Nina y CANT, Sarah (2004). The Hebden Bridge Sculpture Trail: can a collaborative and community arts event stimulate new understandings of landscape, place and identity? Edimburgo. Conference proceedings, OPENspace/Peoplespace.

PECK, Jamie (2005). «Struggling with the creative class». International Journal of Urban and Regional Research, 29 (4), 740-770.

PuTNAM, Robert (2001). Bowling alone: The collapse and revival of American community. Nueva York: Simon and Schuster.

- (2007). «E Pluribus Unum: Diversity and Community in the Twenty-first Century». Scandinavian Political Studies, 30 (2), 137-174.

Ragin, Charles (1987). The Comparative Method. Moving Beyond Qualitative and Quantitative Strategies. Berkeley: University of California Press.

RuIZ, Janet (2004). A Literature Review of the Evidence Base for Culture, the Arts and Sport Policy. Scottish Executive Education Department. 
Simons, Derek y DANG, Steven R. (2006). International Perspectives on Cultural Indicators: A review and compilation of cultural indicators used in selected projects. Centre of Expertise on Culture and Communities / Creative City Network of Canada.

SMITH, Peter (1995). «On the unintended consequences of publishing performance data in the public sector». International Journal of Public Administration, 18 (2/3), 277-310.

STANLEY, Dick (2004). Report of the proceedings of the IFACCA/ISSEC research workshop Looking for new connections. Montreal, August 24-25, 2004. Ottawa.

- (2006). "Introduction: the social effects of culture». Canadian Journal of Communication, 31, 7-15.

UNESCO (1982). Declaración de México. Conferencia mundial sobre políticas culturales.

YPRODUCTIONS (2008). Innovación y creatividad social: Sobre los beneficios económicos de la creatividad de masas (un nuevo marco de explotación). Yproductions eds (edición 2008). [http://ypsite.net/pdfs/innovacionSocial_YP.pdf, consulta: 16 de abril de 2009] 